ELECTRONIC RESEARCH ANNOUNCEMENTS OF THE AMERICAN MATHEMATICAL SOCIETY

Volume 6, Pages 95-97 (October 10, 2000)

S $1079-6762(00) 00083-4$

\title{
THE FLOW COMPLETION OF A MANIFOLD WITH VECTOR FIELD
}

\author{
FRANZ W. KAMBER AND PETER W. MICHOR
}

(Communicated by Alexandre Kirillov )

\begin{abstract}
For a vector field $X$ on a smooth manifold $M$ there exists a smooth but not necessarily Hausdorff manifold $M_{\mathbb{R}}$ and a complete vector field $X_{\mathbb{R}}$ on it which is the universal completion of $(M, X)$.
\end{abstract}

1. Theorem. Let $X \in \mathfrak{X}(M)$ be a smooth vector field on a (connected) smooth manifold $M$.

Then there exists a universal flow completion $j:(M, X) \rightarrow\left(M_{\mathbb{R}}, X_{\mathbb{R}}\right)$ of $(M, X)$. Namely, there exists a (connected) smooth not necessarily Hausdorff manifold $M_{\mathbb{R}}$, a complete vector field $X_{\mathbb{R}} \in \mathfrak{X}\left(M_{\mathbb{R}}\right)$, and an embedding $j: M \rightarrow M_{\mathbb{R}}$ onto an open submanifold such that $X$ and $X_{\mathbb{R}}$ are $j$-related: $T j \circ X=X_{\mathbb{R}} \circ j$. Moreover, for any other equivariant morphism $f:(M, X) \rightarrow(N, Y)$ for a manifold $N$ and a complete vector field $Y \in X(N)$ there exists a unique equivariant morphism $f_{\mathbb{R}}:\left(M_{\mathbb{R}}, x_{\mathbb{R}}\right) \rightarrow$ $(N, Y)$ with $f_{\mathbb{R}} \circ j=f$. The leaf spaces $M / X$ and $M_{\mathbb{R}} / X_{\mathbb{R}}$ are homeomorphic.

Proof. Consider the manifold $\mathbb{R} \times M$ with coordinate function $s$ on $\mathbb{R}$, the vector field $\bar{X}:=\partial_{s} \times X \in \mathfrak{X}(\mathbb{R} \times M)$, and let $M_{\mathbb{R}}:=\mathbb{R} \times{ }_{\bar{X}} M$ be the orbit space (or leaf space) of the vector field $\bar{X}$.

Consider the flow mapping $\mathrm{Fl}^{\bar{X}}: \mathcal{D}(\bar{X}) \rightarrow \mathbb{R} \times M$, given by $\mathrm{Fl}_{t}^{\bar{X}}(s, x)=$ $\left(s+t, \mathrm{Fl}_{t}^{X}(x)\right)$, where the domain of definition $\mathcal{D}(\bar{X}) \subset \mathbb{R} \times(\mathbb{R} \times M)$ is an open neighbourhood of $\{0\} \times(\mathbb{R} \times M)$ with the property that $\mathbb{R} \times\{x\} \cap \mathcal{D}(\bar{X})$ is an open interval times $\{x\}$.

For each $s \in \mathbb{R}$ we consider the mapping

$$
j_{s}: M \stackrel{\text { ins }_{t}}{\longrightarrow}\{s\} \times M \subset \mathbb{R} \times M \stackrel{\pi}{\longrightarrow} \mathbb{R} \times \overline{\bar{X}} M=M_{\mathbb{R}} .
$$

Each mapping $j_{s}$ is injective: A trajectory of $\bar{X}$ can meet $\{s\} \times M$ at most once since it projects onto the unit speed flow on $\mathbb{R}$.

Obviously, the image $j_{s}(M)$ is open in $M_{\mathbb{R}}$ in the quotient topology: If a trajectory hits $\{s\} \times M$ in a point $(s, x)$, let $U$ be an open neighborhood of $x$ in $M$ such that $(-\varepsilon, \varepsilon) \times(s-\varepsilon, s+\varepsilon) \times U \subset \mathcal{D}(\bar{X})$. Then the trajectories hitting $(s-\varepsilon, s+\varepsilon) \times U$ fill a flow invariant open neighborhood which projects on an open

Received by the editors July $27,2000$.

2000 Mathematics Subject Classification. Primary 37C10, 57R30.

Key words and phrases. Flow completion, non-Hausdorff manifolds.

Supported by Erwin Schrödinger International Institute of Mathematical Physics, Wien, Austria. FWK was supported in part by The National Science Foundation under Grant No. DMS9504084. PWM was supported by 'Fonds zur Förderung der wissenschaftlichen Forschung, Projekt P 14195 MAT'. 
neighborhood of $j_{s}(x)$ in $M_{\mathbb{R}}$ which lies in $j_{s}(M)$. This argument also shows that $j_{s}$ is a homeomorphism onto its image in $M_{\mathbb{R}}$.

Let us use the mappings $j_{s}: M \rightarrow M_{\mathbb{R}}$ as charts. The chart change then looks as follows: For $r<s$ the set $\left(j_{s}\right)^{-1}\left(j_{r}(M)\right) \subset M$ is just the open subset of all $x \in M$ such that $[0, s-r] \times\{(s, x)\} \subset \mathcal{D}(\bar{X})$, and $\left(j_{s}\right)^{-1} \circ j_{r}$ is given by $\mathrm{Fl}_{s-r}^{X}$ on this set. Thus the chart changes are smooth.

Consider the flow $(t,(s, x)) \mapsto(s+t, x)$ on $\mathbb{R} \times M$ which commutes with the flow of $\bar{X}$ and thus induces a flow on the leaf space $M_{\mathbb{R}}=\mathbb{R} \times_{\bar{X}} M$. Differentiating this flow we get a vector field $X_{\mathbb{R}}$ on $M_{\mathbb{R}}$.

The construction $(M, X) \mapsto\left(M_{\mathbb{R}}, X_{\mathbb{R}}\right)$ is a functor from the category of smooth Hausdorff manifolds with vector-fields and smooth mappings intertwining the vector fields into the category of possibly non-Hausdorff manifolds with complete smooth vector fields and smooth mappings intertwining these fields. For a pair $(M, X)$ with $X$ a complete vector field the flow completion $\left(M_{\mathbb{R}}, X_{\mathbb{R}}\right)$ is equivariantly diffeomorphic to $(M, X)$ since then any of the charts $j_{s}: M \rightarrow M_{\mathbb{R}}$ is also surjective. From this the universal property follows.

2. Example. Let $(M, X)=\left(\mathbb{R}^{2} \backslash\{0\}, \partial_{x}\right)$. The trajectories of $X$ on $M$ and of $\bar{X}$ on $\mathbb{R} \times M$ in the slices $y=$ constant for $y=0$ and $y \neq 0$ then look as follows:

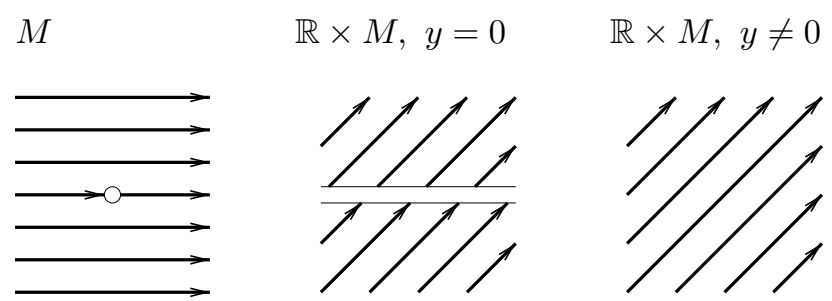

The smooth manifold $M_{\mathbb{R}}$ then is $\mathbb{R}^{2}$ with the $x$-axis doubled: $(x, 0)_{+}$and $(x, 0)_{-}$ cannot be separated for each $x \in \mathbb{R}$. The charts $j_{s}(M)$ all are diffeomorphic to $M=\mathbb{R}^{2} \backslash\{0\}$ and contain $(x, 0)_{-}$for $x<0$ and $(x, 0)_{+}$for $x>0$. The charts $j_{r}(M)$ and $j_{s}(M)$ are glued together by the shift $x \mapsto x+s-r$. In this example $M_{\mathbb{R}}$ is not Hausdorff, but its Hausdorff quotient (given by the equivalence relation generated by identifying non-separable points) is again a smooth manifold and has the universal property described in 1.

3. Example. Let $(M, X)=\left(\mathbb{R}^{2} \backslash\{0\} \times[-1,1], \partial_{x}\right)$. The trajectories of $\bar{X}$ on $\mathbb{R} \times M$ in the slices $y=$ constant for $|y| \leq 1$ and $|y| \geq 1$ then look as in the second and third illustration above. The flow completion $M_{\mathbb{R}}$ then becomes $\mathbb{R}^{2}$ with the part $\mathbb{R} \times[-1,1]$ doubled and the topology such that the points $(x,-1)_{-}$and $(x,-1)_{+}$ cannot be separated as well as the points $(x, 1)_{-}$and $(x, 1)_{+}$. The flow is just $(x, y) \rightarrow(x+t, y):$

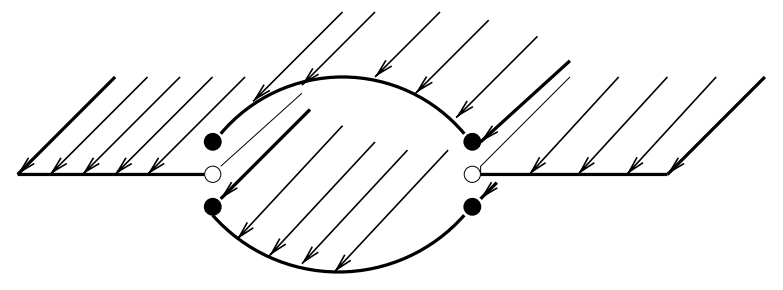


In this example $M_{\mathbb{R}}$ is not Hausdorff, and its Hausdorff quotient is not a smooth manifold any more. There are two obvious quotient manifolds which are Hausdorff, the cylinder and the plane. Thus none of these two has the universal property of 1 .

4. Non-Hausdorff smooth manifolds. We met second countable smooth manifolds which need not be Hausdorff. Let us discuss a little their properties. They are $T_{1}$, since all points are closed; they are closed in a chart. The construction of the tangent bundle is by glueing the local tangent bundles. Smooth mappings and vector fields are defined as usual: non-separable pairs of points are mapped to non-separable pairs. Vector fields admit flows as usual: these are given locally in the charts and are then glued together. If $x$ and $y$ are non-separable points and $X$ is a vector field on the manifold, then for each $t$ the points $\mathrm{Fl}_{t}^{X}(x)$ and $\mathrm{Fl}_{t}^{X}(y)$ are non-separable. Theorem 1 can be extended to the category of not necessarily Hausdorff smooth manifolds and vector fields, without any change in the proof.

5. Remark. The ideas in this paper generalize to the setting of $\mathfrak{g}$-manifolds, where $\mathfrak{g}$ is a finite dimensional Lie group. Let $G$ be the simply connected Lie group with Lie algebra $\mathfrak{g}$. Then one may construct the $G$-completion of a non-complete $\mathfrak{g}$-manifold. There are difficulties with the property $T_{1}$, not only with Hausdorff. This was our original road which was inspired by [1. We treat the full theory in 2]. We thought that the special case of a vector field is interesting in its own.

\section{REFERENCES}

[1] D. V. Alekseevsky and Peter W. Michor, Differential geometry of $\mathfrak{g}$-manifolds., Differ. Geom. Appl. 5 (1995), 371-403, math.DG/9309214. MR 96k:53035

[2] F. W. Kamber and P. W. Michor, Completing Lie algebra actions to Lie group actions, in preparation.

Department of Mathematics, University of Illinois, 1409 West Green Street, UrBANA, IL 61801

E-mail address: kamber@math.uiuc.edu

Institut für Mathematik, Universität Wien, Strudlhofgasse 4, A-1090 Wien, Austria; and: Erwin Schrödinger Institut für Mathematische Physik, Boltzmanngasse 9, A-1090 Wien, Austria

E-mail address: michor@pap.univie.ac.at 